\title{
The stellar mass-metallicity relations of galaxies from high- $z$ to the local
}

\author{
Y. C. Liang ${ }^{1}$, F. Hammer ${ }^{2}$ and L. C. Deng ${ }^{1}$ \\ ${ }^{1}$ National Astronomical Observatories, Chinese Academy of Sciences, 20A Datun Road, \\ Chaoyang District, Beijing 100012, China; email: ycliang@bao.ac.cn \\ ${ }^{2}$ GEPI, Observatoire de Paris-Meudon, 92195 Meudon, France
}

\begin{abstract}
The stellar mass-metallicity relations of star-forming galaxies at intermediate- $z$ $(0.4<z<1)$ and high- $z(z \geqslant 2)$ are compared with the local SDSS galaxies. It shows obvious evolutionary effect, i.e., at a given stellar mass, the intermediate- $z$ galaxies show about 0.3 dex lower metallicity, and the high- $z$ galaxies show about 0.56 dex more metal-deficient than the local ones. These distant galaxies will produce these important parts of their metals during the consequent evolutionary process. A close-box model can explain this evolutionary process generally.
\end{abstract}

Keywords. galaxies: abundances, evolution, high-redshift, ISM, spiral, starburst

Chemical enrichment is a record of star formation history, and the stellar mass provides a more straight-forward measure of the accumulated stellar components inside the galaxies. The correlations between luminosity and metallicity or mass and metallicity of galaxies has been established in nearby and distant galaxies, ranging over orders of magnitude in mass and luminosity and spanning $\sim 2$ dex in chemical abundance. Stellar mass is a more robust indicator for the stellar assembly of galaxies than luminosity since the latter one is more sensitive to the instantaneous star forming. The K-band magnitude is a good indicator for the stellar mass since it is less sensitive to the dust extinction and most related to the stellar components. Another robust method to estimate the stellar masses of galaxies is to fit their SED within a wide spectral coverage by using stellar evolutionary synthesis models, such as Bruzual \& Charlot (2003, MNRAS, 344, 1000).

To understand the assembly history of the stellar masses of galaxies, we compare the M-Z relations of the galaxies at intermediate- $z$ and high- $z$ with the local star-forming galaxies from the SDSS database. For the intermediate- $z$ samples, we select 34 galaxies from the CFRS and Marono fields from Liang, Hammer, Flores (2006, A\&A, 447, 113), and 24 galaxies from the Gemini Deep Deep Survey from Savaglio et al. (2005, ApJ, 635, 260). For the high- $z$ star-forming galaxies with $z \geqslant 2$, we select 7 samples from Shapley et al. (2004, ApJ, 612, 108) and 6 "mean objects" from Erb et al. (2006, ApJ, 644, 813) which are the mean results in the 6 mass bins for their total 87 rest-frame UV-selected galaxies. The local SDSS galaxies are taken from Tremonti et al. (2004, ApJ, 613, 898). The stellar masses of all the samples have been corrected to the same IMF (Salpeter 1955, ApJ, 121, 161) and cosmological model $\left(H_{0}=70 \mathrm{~km} \mathrm{~s}^{-1}\right)$.

The careful comparison show that, at a given stellar mass, the intermediate- $z$ galaxies show about 0.3 dex lower metallicity, and the high- $z$ galaxies show about 0.56 dex more metal-deficient than the local ones. These distant galaxies will produce these most important parts of their metals during the consequent evolutionary process. A close-box model can explain this evolutionary process generally. It leads to a gas fraction converted into stars of $20-25 \%$ since $\mathrm{z} \sim 0.65$ and of $30-37 \%$ since $\mathrm{z} \sim 2.3$ if the gas fraction is $20-10 \%$ remained in the present-day galaxies with intermediate masses. 